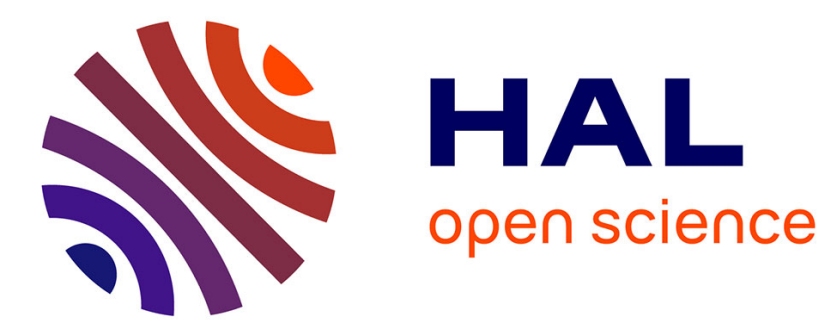

\title{
Segmentation of tumor vessels based on parallel double snakes including region information
}

Pierre Glanc, Florence Rossant, Valérie Rouffiac, Corinne Laplace-Builhé, Ingrid Leguerney, Stéphanie Pitre-Champagnat

\section{To cite this version:}

Pierre Glanc, Florence Rossant, Valérie Rouffiac, Corinne Laplace-Builhé, Ingrid Leguerney, et al.. Segmentation of tumor vessels based on parallel double snakes including region information. IPTA, Nov 2015, Orléans, France. pp.174 - 179, 10.1109/IPTA.2015.7367121 . hal-01567285

\author{
HAL Id: hal-01567285 \\ https://hal.science/hal-01567285
}

Submitted on 15 Dec 2018

HAL is a multi-disciplinary open access archive for the deposit and dissemination of scientific research documents, whether they are published or not. The documents may come from teaching and research institutions in France or abroad, or from public or private research centers.
L'archive ouverte pluridisciplinaire HAL, est destinée au dépôt et à la diffusion de documents scientifiques de niveau recherche, publiés ou non, émanant des établissements d'enseignement et de recherche français ou étrangers, des laboratoires publics ou privés. 


\title{
Segmentation of Tumor Vessels based on Parallel Double Snakes Including Region Information
}

\author{
Pierre Glanc ${ }^{1}$, Florence Rossant ${ }^{1}$, Valérie Rouffiac ${ }^{2}$, \\ Corinne Laplace-Builhé ${ }^{2}$, Ingrid Leguerney ${ }^{3}$ and Stéphanie Pitre-Champagnat ${ }^{4}$ \\ ${ }^{1}$ Institut Supérieur d'Électronique de Paris, 10 rue de Vanves 92130 Issy-les-Moulineaux \\ e-mail: pierre.glanc@isep.fr, florence.rossant@isep.fr \\ 2 Gustave Roussy, Imaging and Cytometry Platform, UMS AMMICA \& Laboratoire IR4M, Université Paris Sud, CNRS, \\ 114 rue Edouard Vaillant 94805 Villejuif Cedex \\ e-mail: valerie.rouffiac@gustaveroussy.fr, corinne.laplace@gustaveroussy.fr \\ ${ }^{3}$ Gustave Roussy \& Laboratoire IR4M, Université Paris Sud, CNRS, 114 rue Edouard Vaillant 94805 Villejuif Cedex \\ e-mail: ingrid.leguerney@gustaveroussy.fr \\ ${ }^{4}$ Laboratoire IR4M, Université Paris Sud, CNRS, 114 rue Edouard Vaillant 94805 Villejuif Cedex \\ e-mail: stephanie.pitre@u-psud.fr
}

\begin{abstract}
In this paper, we address the problem of the segmentation of vessels in images of mouse tumors, with an efficient algorithm that minimizes the user's intervention. For each vessel, two points delimiting its extremities have to be selected. Then, a line inside the vessel is automatically determined based on a Dijkstra-type algorithm. Finally, an original active contour model combining both parallel double snakes and region criteria aims at finding the borders of the vessel. Our segmentation algorithm provides numerical models of tumor vessels, suitable for the simulation of blood and contrast agent flow.
\end{abstract}

Keywords - Image segmentation, Tumor vessels, Active contour models, Parallel Double Snakes, Region criteria.

\section{INTRODUCTION}

Functional imaging of tumor microvasculature is a major challenge in oncology to assess early therapeutic efficacy of new drugs which target vessel destruction. This imaging method aimed at detecting microcirculation modifications prior to change of tumor volume. Specifically, it is based on the study of microvascular enhancement by injection of contrast agents (CA) for characterizing the microcirculation (mainly blood volume and blood flow).

The recent introduction of microbubbles as CA has allowed access to ultrasound functional imaging of tumor microvascularization. This new modality called Dynamic ContrastEnhanced Ultrasonography (DCE-US) has been validated by a multicenter clinical study [11] and is now included in the European and International guidelines. However, DCEUS remains a recent functional imaging modality and clinical research indicates the necessity to master the variability of the quantification methods of tumor perfusion. To achieve this task, we propose to characterize these quantification methods through a numerical modeling of the flow of ultrasound CA [2]. This requires realistic digital phantoms of vascular networks, approximating the tumorous physiological conditions. Our approach consists in designing these phantoms from sequences of images of in-vivo vascular networks, acquired at regular time step and segmented by dedicated image processing algorithms.
This paper presents the methods we propose to perform the segmentation of mouse tumor vessels from images acquired in macro-fluorescence and confocal microscopy (Fig. 1), in order to provide realistic $2 \mathrm{D}+\mathrm{t}$ models.

Many approaches have been proposed for the segmentation of vessels (retina, coronary artery, etc.) in many imaging modalities, see for example the review in [13]. They exploit the panel of tools available in the image processing field and adapt them to the application specificity: vessel enhancement based on wavelets [21], morphological filter [24], Hessianbased filters [1], [22], [14], pixel classification [22], [21], region growing algorithms [9], [14], [16], tracking procedures [8], active contour models [15], [12], etc. However, to the best of our knowledge, very little work has been dedicated to the analysis of tumor vessels. For example, the authors of [25], [7], [19]. proposed image processing methods in order to quantify the tumor angiogenesis, by estimating the vessel branch density and length, but no accurate segmentation of the vessel branches is performed.

The tumor vasculature is known to be particularly tortuous and irregular, including shunts and loops, responsible for the complexity of the microcirculation mechanisms. A fully automatic process seems to be very difficult to achieve. That is why we propose a semi-automatic procedure, based on minimal path techniques combined with active contour models. Indeed, these tools have proven to be powerful, even for processing complex images, and are well suited to approaches guided by user.
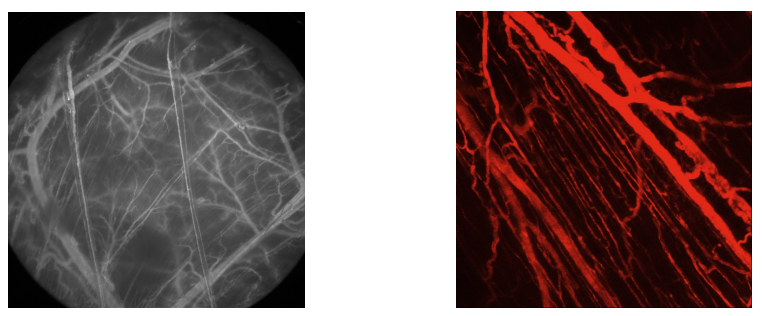

Fig. 1. A macro fluorescence (left) and a confocal (right) images. 
Active contour techniques are based on the minimization of an energy functional and were originally introduced by [10]. The ISEP team has developed recently a new model, the Parallel Double Snakes, that incorporates a parallelism constraint in the energy function, allowing the simultaneous extraction of two almost parallel contours [20]. It has been succesfully applied for the segmentation of vessels in adaptive optics [12]. This approach can be coupled with a centerline extraction method [4], [5], in order to initialize the snakes. Data finally obtained are composed of a centerline combined with measures of local radii and seem to be perfectly adapted to the digital model required by the software for flow modeling.

Other active contours method based on region criteria have been developed [18]. As they do not focus on gradients, they may better handle images with low contrast.

The two latter approaches appeared useful to us for processing our sequences of images containing very heterogeneous vessels. Consequently, we focus in this paper on an original method combining both approaches: after the extraction of the centerline, we minimize an energy that adds up gradient- and region-based terms.

In a first part, we present the main features of images we are dealing with, before exposing the algorithm in detail: determination of vessel centerlines in a first part, parallel double snakes with region criteria in a second part. We end this paper with experimental results including quantitative evaluation.

\section{IMAGES WE FOCUS ON}

\section{A. Image features}

The images we consider in the paper are composed of two sets, depending on the acquisition device (see Fig. 1).

The first set is composed of macro fluorescence images with resolution $11.5 \mu \mathrm{m} /$ pixel (left image of Fig. 1). In this case, we deal with a great part of the vascular network. Borders of tumor vessels appear very irregular, as compared to other segmentation problems of tubular structures, making their localization very challenging. Noticeably, size, curvature and contrast of the vessels are very heterogeneous (see also the left image of Fig. 2, where we zoomed on the tumor on another macro fluroescence image), which implies to develop a robust method to address all different configurations.

The second set is made up of the so-called confocal images with resolution $2.9 \mu \mathrm{m} /$ pixel (right in Fig. 1). They are obtained by scanning the tumor zone in depth and by adding up the acquired slices. They focus on small details of the network and give complementary information to the macro fluorescence images. Features of vessels in these images are quite different from those of macro fluorescence images. Indeed, curvatures are lower, but vessels are even more irregular, with important variations in intensity (see the right image of Fig. 2).
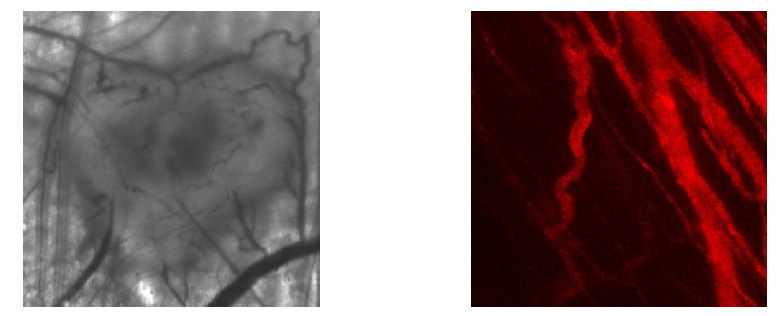

Fig. 2. Zoom on the tumor region of a macro fluorescence (left) and a confocal (right) images.

\section{B. Pretreatments}

The first step of the algorithm aims at enhancing the vessels and improving the signal to noise ratio. We apply a Top-Hat transform with circular structuring elements. This processing extract narrow intensity peaks (narrower than the structuring element). As the diameter of most vessels is comprised between 1 and 15 pixels, we chose radii in the interval $[1,15]$ and average the obtained images.

In the case of confocal imaging, we finish this preprocessing step by an opening of the image with a circular structuring element of size 3 in order to reduce the remaining noise.

Figure 3 shows the results of these pretreatments from the images of Fig. 1.
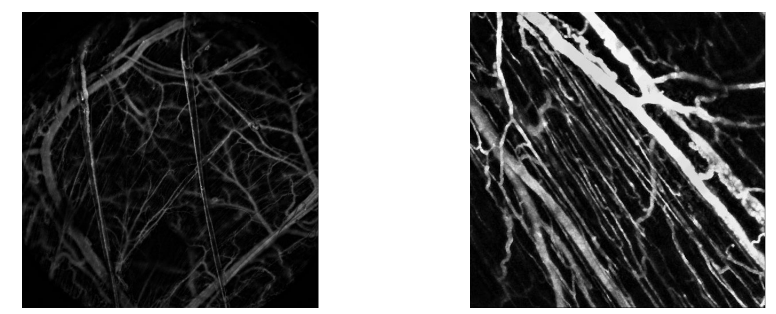

Fig. 3. Images after preprocessing.

\section{Determining the VESSEL CENTERLINE}

At the end of our global algorithm, we aim at obtaining a suitable representation of the vessel branches, made up of the discrete representation of their centerline combined with the measure of the local radius at every point. This mode is suitable for both segmentation algorithms and numerical models involved in the simulation of the fluid flow. Thus, the first step consists in determining a median line lying inside every vessel branch, the extremities having been previously pointed by the user.

\section{A. Requirements}

We use the classical point of view that considers pixels $p$ of the image $I$ as vertices of a graph. With this formalism, determining the centerline of a vessel consists in finding the minimal path in this graph between the two extremities of the vessel thanks to Dijkstra's algorithm [6]. The key point is to find the adequate cost attached to the edges of the graph.

We want the centerline to fulfill three properties: 
- The centerline should stay inside the vessel.

- The centerline should be located as much as possible in the "middle" of the vessel, and not be "glued" to its boundaries.

- The centerline should be as regular as possible, in other terms, free of undesirable "zigzags".

\section{B. Ponderation of the graph edges}

1) First condition: In the Dijkstra's formalism, the minimal path in a graph is the one that minimizes the sum of the edge path costs. Thus, we must encourage the edges that tend to follow the previous properties and penalize the others. To illustrate this, let us consider two neighbor pixels $p_{1}$ and $p_{2}$ of our graph, and the cost of the corresponding edge: $P\left(p_{1}, p_{2}\right)$.

Let $p_{0}$ be the source pixel, i.e. one of the two extremities selected by the user. The intensity inside the vessel is not constant but nevertheless quite homogeneous and brighter than the background. It should also be close to the image intensity at $p_{0}$. Thus we initially choose:

$$
P\left(p_{1}, p_{2}\right)=\left[\frac{I\left(p_{1}\right)+I\left(p_{2}\right)}{2}-I\left(p_{0}\right)\right]^{2},
$$

where $I(p)$ is the intensity at pixel $p$.

2) Second condition: The previous cost is not completely satisfactory as the resulting centerline often follows one of the vessel boundaries, as "glued" on it. To solve this problem, we filter $I$ by a Gaussian filter $(\sigma=5)$ and replace $I$ by $\tilde{I}$, the filtered image, in (1). Indeed, the brightest pixels in $\tilde{I}$, which are also the ones whose values are the closest to $I\left(p_{0}\right)$, are located "in the middle" of the vessel.

$$
P\left(p_{1}, p_{2}\right)=\left[\frac{\tilde{I}\left(p_{1}\right)+\tilde{I}\left(p_{2}\right)}{2}-I\left(p_{0}\right)\right]^{2} .
$$

3) Third condition: Finally, the third of the previous properties seems to be the most straightforward, and can be reformulated in these terms: the two other properties being satisfied, we want the centerline to be as small as possible. So, we introduce the euclidian distance $d\left(p_{1}, p_{2}\right)$, between $p_{1}$ and $p_{2}$ as follows:

$$
P\left(p_{1}, p_{2}\right)=\gamma d\left(p_{1}, p_{2}\right)+\zeta\left[\frac{\tilde{I}\left(p_{1}\right)+\tilde{I}\left(p_{2}\right)}{2}-I\left(p_{0}\right)\right]^{2},
$$

$\gamma$ and $\zeta$ being weighting parameters. In practice, we choose $\gamma=0.01$ and $\zeta=1$.

\section{FINDING THE VESSEL BORDERS: PARALLEL DOUBLE SNAKES WITH REGION CRITERIA}

\section{A. Parallel Double Snakes}

Once we have found the centerline of vessels, the next step is to determine their boundaries. An efficient method has been developed in [20] for the segmentation of elongated features such as vessels. It is based on the simultaneous evolution of two almost parallel lines (snakes) through the minimization of a gradient-based energy. We now remind the key points of this method.
Let us denote by $V(s), s \in[0,1]$, the reference line of the PDS model (in practice, initialized as the centerline found previously), parameterized by the curvilinear abscissa $s$. Let us also introduce two other curves, $V_{1}$ and $V_{2}$, defined with respect to $V$ by: $V_{1,2}(s)=V(s) \pm b(s) n(s)$, where $n(s)$ is the vector normal to $V(s)$ and $b(s)$ the local radius.

The energy to be minimized is defined as follows:

$$
E(V, b)=E_{\text {image }}\left(V_{1}, V_{2}\right)+E_{\text {int }}(V)+R\left(V_{1}, V_{2}\right),
$$

where:

$$
\begin{gathered}
E_{\text {image }}\left(V_{1}, V_{2}\right)=-\int_{0}^{1}\left[\left|\nabla I\left(V_{1}(s)\right)\right|^{2}+\left|\nabla I\left(V_{2}(s)\right)\right|^{2}\right] d s \\
E_{\text {int }}(V)=\frac{1}{2} \int_{0}^{1} \alpha(s)\left|\frac{\partial V(s, t)}{\partial s}\right|^{2}+\beta(s)\left|\frac{\partial^{2} V(s, t)}{\partial s^{2}}\right|^{2} d s \\
R\left(V_{1}, V_{2}\right)=\int_{0}^{1} \phi(s)\left(b^{\prime}(s)\right)^{2} d s
\end{gathered}
$$

$\alpha(s), \beta(s)$ and $\phi(s)$ being weighting parameters.

(4) is composed of three terms: the first two ones (5), (6) are classical and aim at driving the curves $V_{1}$ and $V_{2}$ to the edges while preventing them to be too much tortuous. The last one is a coupled energy term, that controls the parallelism constraint between $V_{1}$ and $V_{2}$ : the higher it is, the more strict is the parallelism constraint. This model introduces contextual information about the shape of the area to be segmented, making the snakes more robust to noise, lack of contrast and approximate initialization [20], [12]. It is well suited to our problem.

In practice, we noticed that the weighting $\beta$ is not important as compared to the influence of $\alpha$, and that $\phi$ can be constant along the snake. So we set $\beta=0$ for all images, $\phi=700$ (quite strong parallelism constraint) for the macro fluorescence images and $\phi=20$ for the confocal images. On the contrary, it is necessary to make $\alpha(s)$ vary as a function of $s$ since vessel branches can exhibit both straight and strongly bent parts. Figure 4 illustrates this point: when $\alpha$ is uniform on the whole vessel, either the twist is bad captured (case $\alpha$ big) or the snake is too much tortuous (case $\alpha$ small). That is why we propose an original method for calculating automatically this weighting parameter from the centerline features.
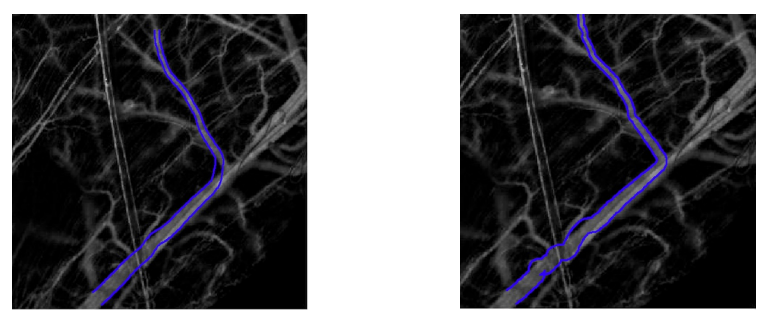

Fig. 4. Left: segmentation with $\alpha=10$, right: segmentation with $\alpha=1$.

\section{B. Determination of $\alpha(s)$}

Consider the left scheme of Fig. 5 where $p_{1}, p_{2}$ and $p_{3}$ are three points of the centerline and $d_{1}$ and $d_{3}$ are the distances 
between $p_{2}$ and $p_{1}$, and $p_{2}$ and $p_{3}$, respectively. We want to determine the value of $\alpha$ at point $p_{2}$. Our method is based on an estimation of $\cos \left(\hat{p}_{2}\right)$, normalized to give a measure $c$ in the interval $[0,1]$ :

$$
c=\frac{\overrightarrow{p_{2} p_{1}} \cdot \overrightarrow{p_{2} p_{3}}+d_{1} d_{3}}{2 d_{1} d_{3}} .
$$

$c \simeq 0$ for approximately aligned points, while $c$ tends to 1 for sharp angles. The bigger $\alpha$ is, the more the snake is rigid. Thefore, we compute $\alpha\left(p_{2}\right)$ with the following formula (see the graph at the right-hand side of Fig. 5):

$$
\alpha\left(p_{2}\right)=10(1-c)^{10} .
$$
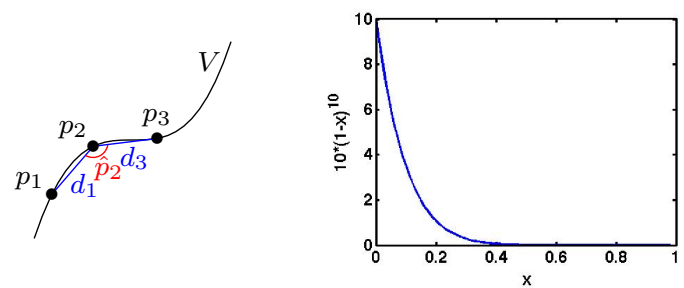

Fig. 5. Determination of $\alpha$. Left: notations used; right: plot of the function $c \mapsto 10(1-c)^{10}$ on $[0,1]$.

We choose a fast decreasing function as we want the snake to become rapidly less rigid as $\hat{p}_{2}$ passes from $\pi$ to smaller angles values: $\alpha \simeq 0$ when $\hat{p}_{2}<2 \pi / 3$,

In practice, we define a fixed step $S$ for all vessels of the image so that, $p_{1}=V_{i}, p_{2}=V_{i+S / 2}$ and $p_{3}=V_{i+S}$, where $V_{i}=V(s=i h)$ denotes the discretized centerline $V(N$ points), $h=1$ and $i \in[1, N-S]$. We take $S=100$, which allows us to adapt the weighting parameter according to the significant variation of the vessel curvature. When the size of the vessel is below $S, \alpha$ remains the same at every point of the snake. It is computed using the two extremities of the centerline and the point at the middle of it.

\section{Region criteria}

The previous method provides good results when the contrast between the vessels and the background is good. Unluckily, this is not always the case, even after the pretreatments. For this reason, a region-oriented criterion has been integrated in the PDS model.

In [17], [18], a model has been developed where zones to be segmented have to verify a homogeneity criterion. This method is inspired by the Chan and Vese approach [3], but is adapted to the segmentation of tubular structures.

Two regions are defined (Fig. 6):

- For the first one, $R_{i n}$, the authors use the same formalism as ours in the PDS model: $R_{i n}$ is the region localized "inside" the curves $V_{1}$ and $V_{2}$.

- A second region, $R_{\text {out }}$ is defined by considering two additional curves forming an outside band with a constant thickness $B$.

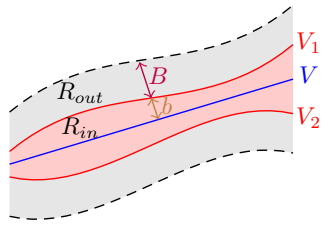

Fig. 6. Regions $R_{i n}$ and $R_{\text {out }}$ considered in the model including a region criterion.

With this formalism, a region-based energy is defined by:

$$
E_{\text {region }}\left(V_{1}, V_{2}\right)=\int_{R_{\text {in }}} g_{\text {in }}(\mathbf{x}) d \mathbf{x}+\int_{R_{\text {out }}} g_{\text {out }}(\mathbf{x}) d \mathbf{x},
$$

where region descriptors $g_{\text {in }}$ and $g_{\text {out }}$ penalize the intensity heterogeneity in the the two defined bands.

The authors have shown in [18] that minimizing the energy (10) can be explicitly formalized as a function of $V$ and $b$, which enables us to easily combine this approach with the PDS model.

\section{Complete energy computation}

We finally define the following complete energy:

$$
\begin{aligned}
& E(V, b)=\psi E_{\text {image }}\left(V_{1}, V_{2}\right)+\delta E_{\text {region }}\left(V_{1}, V_{2}\right) \\
& +E_{\text {int }}(V)+R\left(V_{1}, V_{2}\right),
\end{aligned}
$$

with $E_{\text {image }}\left(V_{1}, V_{2}\right), E_{\text {int }}(V)$ and $R\left(V_{1}, V_{2}\right)$ as in (4), $E_{\text {region }}\left(V_{1}, V_{2}\right)$ as in (10), and $\psi$ and $\delta \in[0,1]$ being weighting parameters such that $\psi+\delta=1$.

The minimization is performed by gradient descent, by alternatively minimizing over $V$ and over $b$.

The associated Euler-Lagrange equations are:

$$
\begin{aligned}
& -\alpha(s) V^{\prime \prime}(s)-\alpha^{\prime}(s) V^{\prime}(s)+\beta V^{(4)}(s)-\psi\left[F\left(V_{1}(s)\right)+F\left(V_{2}(s)\right)\right] \\
& +\delta\left[(1-\kappa(s) b(s))\left(g_{\text {in }}\left(V_{1}(s)\right)-g_{\text {out }}\left(V_{1}(s)\right)\right)\right. \\
& \left.-(1+\kappa(s) b(s))\left(g_{\text {in }}\left(V_{2}(s)\right)-g_{\text {out }}(V 2(s))\right)\right] n(s)=0
\end{aligned}
$$

for the minimization of $E$ with respect to $V$, and

$$
\begin{aligned}
& -\phi b^{\prime \prime}(s)+\psi\left[n(s) \cdot\left(F\left(V_{2}(s)\right)-F\left(V_{1}(s)\right)\right)\right] \\
& +\delta\left[(1-\kappa(s) b(s))\left(g_{\text {in }}\left(V_{1}(s)\right)-g_{\text {out }}\left(V_{1}(s)\right)\right)\right. \\
& \left.+(1+\kappa(s) b(s))\left(g_{\text {in }}\left(V_{2}(s)\right)-g_{\text {out }}(V 2(s))\right)\right]=0
\end{aligned}
$$

for the minimization of $E$ with respect to $b$. We have noted here $P=-\|\nabla I\|^{2}, F=-\nabla P$ (or $F$ is derived from the Gradient Vector Flow, see [23]), $\kappa(s)$ the local curvature and we have chosen $g_{\text {in }}$ and $g_{\text {out }}$ like in [17] (based on mean intensity values).

\section{E. Importance of a region criterion for our images}

As we mentioned above, we deal with images whose contrast is not always optimal. In some cases, the algorithm of PDS without region criterion fails at finding a correct vessel segmentation in zones where the contrast is low. Figure 7 shows such a case. On the left, where no region criterion is integrated $(\psi=1$ and $\delta=0)$, the segmentation is not satisfying: the snake even tries to follow another vessel at the top of the image. On the contrary, we set $\psi=0.7$ and $\delta=0.3$ 
for the segmentation on the right: the result is far better. Therefore, the combination of both gradient- and region-based terms provides a better robustness to the initialization.
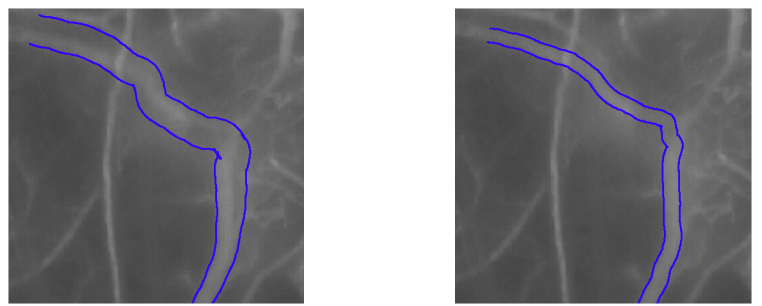

Fig. 7. Integrating a region criterion is the algorithm often improves the quality of the segmentation where the contrast is low.

\section{REsults}

This section is devoted to results obtained with the method exposed in this paper. For several macro fluorescence and confocal images, we have selected some interesting vessels to segment. We present the results obtained with our algorithm and compare them to manual segmentations made by two experts of Institut Gustave Roussy. In all cases, the parameter $b$ is chosen constant equal to 8 at the initialization and we set $B=20, \psi=0.7$ and $\delta=0.3$.

On Fig. 8, we firstly present the results of the segmentation of two vessels in a macro fluorescence image. It is quite blurry and vessels have a relatively high curvatures, but they are quite regular, i.e. without brutal variations of the radius or the curvature. Our algorithm is able to segment these vessels quite easily and the results are very good (on the right-hand side, we put the manual segmentation of an expert, for comparison).
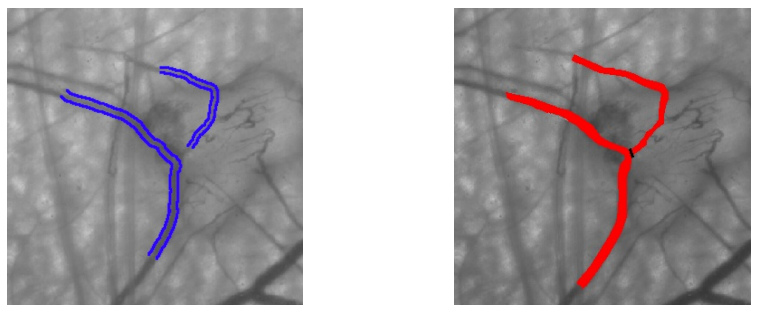

Fig. 8. (Relatively) regular vessels in a macro fluorescence image segmented by our algorithm (left) and by an expert (right).

On Fig. 9, we focus on the zoom of a macro fluorescence image, the one we presented on Fig. 2. Once again, the image is blurry, and this time the vessel on the right of the image is furthermore very irregular and tortuous. Despite this fact, the segmentation obtained with our algorithm is satisfactory. We once again show the importance of the automatic adaptation of the parameter $\alpha$ : the top of the vessel requires to have a very small $\alpha$ while the vertical part can be segmented with greater values. Nevertheless, we notice that the top-right of the vessel is not exactly segmented: a very brutal variation of the local curvature (pointed by the arrow) was not captured, which implies that improvements on the costs of Dijkstra's graph edges or on the active contour parameters can still be done.
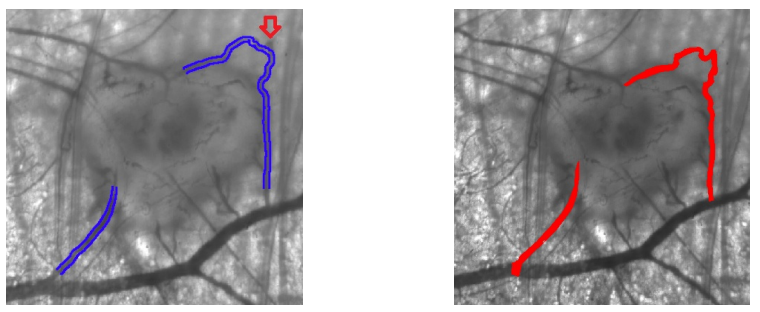

Fig. 9. Two vessels from the macro fluorescence image of Fig. 2, segmented by our algorithm (left) and by an expert (right).

On Fig. 10, we show segmentations of vessels in two confocal images. As we already mentioned, characteristics of these images are different from those of macro fluorescence images: vessels have lower curvatures but have more small irregularities. As a consequence, we chose $\phi$ smaller for these images (see above), which allows the local radius to vary more. All small details are not exactly captured in these cases, but the segmentations obtained are once again satisfactory.
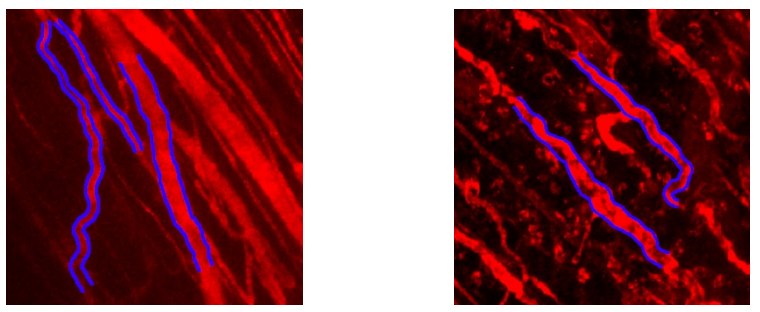

Fig. 10. Vessels in two confocal images segmented by our algorithm.

We now present a table that summarizes mean square errors (MSE) obtained in three cases: the first two are our segmentation compared with the manual one performed by an expert 1 , and with the one made by another expert 2 . To put in perspective the mean square errors values, we also compared the two manual segmentations in order to evaluate the interexperts variability. We selected 8 macro fluorescence images (denoted by "M" in the table), each of them containing two vessels to segment (denoted by "V"). Means and standard deviations for the three cases are also given.

The same work is performed for two confocal images, denoted by "C" in the following table.

In overall, our segmentations are closer to those of expert 2 than to those of expert 1. The auto/experts errors are in the same order of magnitude as the inter-experts ones. These latter are quite high, which confirms the fact that both macro fluorescence and confocal images are quite blurry and hard to interpret. We also notice that some very tortuous vessels are hard to segment (even for the experts): for instance, the image $\mathrm{C} 2$ in the table corresponds to the image on the right-hand side of Fig. 10. On the contrary, a very good accordance is obtained between the semi-automatic and the manual segmentation for more regular vessels. 
Table 1. MSE for eight macro fluorescence images, in pixel unit.

\begin{tabular}{|l|c|c|c|}
\hline & \multicolumn{3}{|c|}{ Mean Square Errors } \\
\cline { 2 - 4 } M1V1 & auto/expert 1 & auto/expert 2 & expert 1/expert 2 \\
M1V2 & 1.86 & 0.87 & 1.81 \\
M2V1 & 1.94 & 1.11 & 1.64 \\
M2V2 & 2.09 & 0.92 & 1.93 \\
M3V1 & 2.36 & 1.05 & 2.88 \\
M3V2 & 5.69 & 1.65 & 5.21 \\
M4V1 & 6.13 & 1.90 & 4.19 \\
M4V2 & 1.57 & 1.11 & 1.79 \\
M5V1 & 2.68 & 2.39 & 3.57 \\
M5V2 & 2.05 & 1.27 & 1.96 \\
M6V1 & 3.54 & 1.66 & 2.44 \\
M6V2 & 1.54 & 0.74 & 1.82 \\
M7V1 & 2.41 & 1.30 & 2.34 \\
M7V2 & 2.12 & 1.64 & 1.61 \\
M8V1 & 2.21 & 1.40 & 1.11 \\
M8V2 & 2.16 & 1.41 & 1.83 \\
\hline Mean & 2.66 & 0.99 & 2.57 \\
\hline SD & 2.69 & 1.34 & 2.42 \\
\hline
\end{tabular}

Table 2. MSE for two confocal images, in pixel unit.

\begin{tabular}{|l|c|c|c|}
\hline \multirow{2}{*}{} & \multicolumn{3}{|c|}{ Mean Square Errors } \\
\cline { 2 - 4 } C1V1 & auto/expert 1 & auto/expert 2 & expert 1/expert 2 \\
\hline C1V2 & 2.22 & 1.80 & 2.55 \\
C1V3 & 1.77 & 0.84 & 1.96 \\
C2V1 & 2.07 & 1.78 & 2.03 \\
C2V2 & 6.19 & 2.31 & 4.24 \\
Mean & 2.05 & 1.23 & 2.12 \\
\hline SD & 2.86 & 1.59 & 2.58 \\
\hline
\end{tabular}

\section{CONCLUSION}

We have developed an original method for the semiautomatic segmentation of mouse tumor vessels. The algorithm requires little intervention of the operator and is composed of two main parts: firstly, the search for vessel centerlines thanks to a Dijkstra's algorithm with adequate edge costs; secondly, parallel double snakes with region criteria that evolve by minimizing an energy based on both gradient and region informations.

The segmentation results enable the physicists of Institut Gustave Roussy to develop simple fluid flow models. It is worth noting that it is unnecessary to get a complete segmentation of the vessel network, since modeling fluid flows is a very complex task: dealing with some (interesting) vessels already provides useful informations for physicists.

Besides improvements of the existing algorithms, we envisage to enhance quality of the images: for instance, work is currently ongoing to optimize dorsal skinfold chambers applied on mice to get the images. This will enable us to deal with images with better quality and thus, to get more complete and more precise segmentations. We also plan to integrate the time dimension in our studies, as it will be possible to segment a given zone at different times.

\section{ACKNOWLEDGMENTS}

This project is granted by the Plan Cancer 2009-2013 of the Institut National de la Santé et de la Recherche Médicale (INSERM, Convention $\mathrm{N}^{\circ} \mathrm{PC} 201330$ ).

\section{REFERENCES}

[1] S. R. Aylward and E. Bullitt. Initialization, noise, singularities, and scale in height ridge traversal for tubular object centerline extraction. IEEE Transactions on Medical Imaging, 21(2): 61-75, 2002.
[2] L. Boyer, P. Le Notre, S. Randall Thomas, I. Leguerney, N. Lassau and S. Pitre-Champagnat. Numerical modelling of the flow of the ultrasound contrast agents in tumour microvasculature. Computer methods in biomechanics and biomedical engineering, 17 Suppl. 1:18-9, 2014.

[3] T. Chan and L. Vese. Active contours without edges. IEEE Transactions on Image Processing, 10(2): 266-277, 2001.

[4] L. Cohen and R. Kimmel. Global minimum for active contour models: a minimal path approach. International Journal of Computer Vision, 24(1): 57-78, 1997.

[5] T. Deschamps and L. D. Cohen. Fast extraction of minimal paths in 3D images and applications to virtual endoscopy. Medical Image Analysis, 5(4): 281-299, 2001.

[6] E. W. Dijkstra. A note on two problems in connexion with graphs Numerische Mathematik, 1(1): 269-271, 1959.

[7] C. N. Doukas, I. Maglogiannis and A. A. Chatziionnou Computersupported angiogenesis quantification using image analysis and statistical averaging. IEEE Transactions on Information Technology in Biomedicine, 12(5): 650-657, 2008.

[8] X. Gao, et al. A method of vessel tracking for vessel diameter measurement on retinal images. In International Conference on Image Processing (ICIP'01), Thessaloniki-Greece, October 2001.

[9] A. Hoover, V. Kouznetsova and M. Golbaum. Locating blood vessels in retinal images by piecewise threshold probing of a matched filter response. IEEE Transactions on Medical Imaging, 19(3): 203-210, 2000.

[10] M. Kass, A. Witkin and D. Terzopoulos. Snakes: Active contour models. International Journal of Computer Vision, 1(4): 321-331, 1988.

[11] N. Lassau, J. Bonastre, M. Kind, V. Vilgrain, J. Lacroix, M. Cuinet, et al. Validation of dynamic contrast-enhanced ultrasound in predicting outcomes of antiangiogenic therapy for solid tumors: the French multicenter support for innovative and expensive techniques study. Investigative Radiology, 49(12): 794-800, 2014.

[12] N. Lermé, F. Rossant, I. Bloch, M. Pâques, E. Koch. Segmentation of retinal arteries in adaptative optics images. In International Conference on Pattern Recognition (ICPR'14), Stockholm-Sweden, August 2014.

[13] D. Lesage, et al. A review of 3D vessel lumen segmentation techniques: models, features and extraction schemes. Medical Image Analysis, 13(6): 819-845, 2009.

[14] M. Martinez-Perez, et al. Retinal blood vessel segmentation by means of scale-space analysis and region growing. In Medical Image Computing and Computer-Assisted Intervention (MICCAIÕ99), Cambridge-United Kingdom, September 1999.

[15] T. McInerney and D. Terzopoulos. T-snakes: topology adaptive snakes. Medical Image Analysis, 4(2): 73-91, 2000.

[16] C. Metz, et al. Semi-automatic coronary artery centerline extraction in computed tomography angiography data. In International Symposium on Biomedical Imaging, Washington-United States, April 2007.

[17] J. Mille and L. Cohen. Reconstruction de structures arborescentes par chemins minimaux et bande déformable. In GRETSI'09, Dijon-France, 2009.

[18] J. Mille, R. Boné and L. D. Cohen. Region-Based 2D Deformable Generalized Cylinder for Narrow Structures Segmentation In 10th European Conference on Computer Vision, Marseille-France, October 2008.

[19] A. Niemisto et al. Robust quantification of in vitro angiogenesis through image analysis. IEEE Transactions on Medical Imaging, 24(4): 549-553, 2005.

[20] F. Rossant, I. Bloch, I. Ghorbel, M. Pâques. Parallel Double Snakes. Application to the segmentation of retinal layers in 2D-OCT for pathological subjects. To appear in Pattern Recognition.

[21] J. V. Soares, et al. Retinal vessel segmentation using the 2-D Gabor wavelet and supervised classification. IEEE Transactions on Medical Imaging, 25(9): 1214-1222, 2006.

[22] J. Staal, et al. Ridge-based vessel segmentation in color images of the retina. IEEE Transactions on Medical Imaging, 23(4): 501-509, 2004.

[23] C. Xu and J. L. Prince. Snakes, shapes and Gradient Vector Flow. IEEE Transactions on Image Processing, 7(3): 359-369, 1998.

[24] F. Zana and J.-C. Klein. Segmentation of vessel-like patterns using mathematical morphology and curvature evaluation. IEEE Transactions on Image Processing, 10(7): 1010-1019, 2001.

[25] B. Zhang et al. AngiolQ: a novel automated analysis approach for angiogenesis image quantification. Biomedical engineering and informatics. In Biomedical Engineering and Informatics (BMEI'09), Tianjin-China, October 2009. 